\title{
Communication environment analysis of textile antenna using ray tracing method
}

\author{
Daisuke Yamanaka $^{1,}$ a) and Masaharu Takahashi $^{1, \text { b) }}$ \\ ${ }^{1}$ Graduate School of Engineering, Chiba University, \\ 1-33 Yayoi-cho, Inage-ku, Chiba-shi, Chiba 263-8522, Japan \\ a)daisuke.yamanaka@m.ieice.org \\ b) omei@faculty.chiba-u.jp
}

Abstract: Biological information monitoring systems are widely used to obtain and monitor the vital signs of patients in hospitals. However, the current system mainly uses the $420-450 \mathrm{MHz}$ band, making it difficult to manage channel switching, adding and removing equipment and so on, unless you are an expert in wireless communications. There is also the problem of having to carry a transmitter that is the same size as a small terminal. Therefore, we proposed a textile antenna using the $5.2 \mathrm{GHz}$ band as a replacement for the system's transmitter antenna. In this paper, we report the antenna characteristics of the transmitter antenna designed by the authors when it is attached to the human body, the received power results of the transmitter power by analyzing the textile antenna in a simulated real-world environment model.

Keywords: ray-tracing method, textile antenna, dual polarized, patch antenna

Classification: Antennas and Propagation

\section{References}

[1] Nihon Kohden Corporation, "Patient monitoring," https://eu.nihonkohden.com/ en/products/patientmonitoring, accessed on: Dec. 18, 2017.

[2] Y. Nakatani and M. Takahashi, "Textile antenna for biological information monitoring," International Symposium on Antennas and Propagation 2016, Okinawa, Japan, pp. 986-987, Oct. 2016.

[3] H.S. Berger and H.M. Gibson, "Managing your hospital RF spectrum," Biomedical Instrumentation \& Technology, vol. 47, no. 3, pp. 193-197, May/June 2013. DOI: $10.2345 / 0899-8205-47.3 .193$

[4] D. Yamanaka and M. Takahashi, "5.2 GHz band textile antenna for biological information monitoring system," 2018 IEEE International Symposium on Antennas and Propagation and USNC-URSI Radio Science Meeting, Boston, U.S., pp. 1295-1296, July 2018. DOI: 10.1109/APUSNCURSINRSM.2018.8609143

[5] D. Yamanaka and M. Takahashi, "5.2 GHz band textile antenna for biological information monitoring," IEICE Trans. Commun. (Japanese Edition), vol. J101B, no. 7, pp. 584-591, July 2018. DOI: 10.14923/transcomj.2017JBP3057

[6] D. Yamanaka and M. Takahashi, "Investigation of the characteristics of a 5.2 GHz textile antenna on a human body," 2019 Wireless Days (WD), Manchester, United Kingdom, pp. 1-4, 2019. DOI: 10.1109/WD.2019.8734212 
[7] T. Nagaoka, S. Watanabe, K. Sakurai, E. Kunieda, S. Watanabe, M. Taki, and Y. Yamanaka, "Development of realistic high-resolution whole-body voxel models of Japanese adult male and female of average height and weight, and application of models to radio-frequency electromagnetic-field dosimetry," Phys. Med. Biol., vol. 49, no. 1, pp. 1-15, Jan. 2004. DOI: 10.1088/0031-9155/49/1/001

\section{Introduction}

It is important to grasp a patient's biological information in a hospital because of the sudden situation change may occur accidentally [1]. For here the vital signs are heart rate, blood pressure, respiratory rate, body temperature and the like. This information has been obtained from various sensors attached to the human body surface, and a transmitter which installed outside the body transmits that information to a bedside monitor or a central monitor at a nurse station [2]. When the transmitter falling beneath the bed would cause an unstable communication problem. The transmitter must always be carried around to satisfies the stable quality of radio communication. Besides, cables connecting the terminal and various sensors interfere with movement. Also, frequencies used in the current system are in the 420 to $450 \mathrm{MHz}$ band, and operations are being administered to avoid interference by assigning and managing channels for each ward [2]. In addition, it is clinical engineering technicians, not radio professionals, who manage the wireless environment in actual medical practice. For them, the specialized tasks of managing channels on a ward-by-ward basis and adding or removing equipment are not easy. That is why we propose the use of wireless LANs as a solution to this problem. Meanwhile, in recent years, congestion and interference of $2.4 \mathrm{GHz}$ band wireless LAN in hospitals have been reported. It is because of the popularization of devices equipped with wireless LAN for medical staff and patients and an increase in medical devices using wireless LAN [3].

However, there are almost no investigations as a single package on the design of an antenna for the off-body network, analysis of characteristics when installed close to the human body, and $5.2 \mathrm{GHz}$ indoor propagation analysis by ray-tracing method when using its antenna for off-body communication.

In our previous study $[4,5]$ and [6], a textile antenna was proposed as a transmitting antenna for a biological monitoring system using the $5.2 \mathrm{GHz}$ band which is not congested [3] show in Fig. 1. In this paper, we report the results of antenna characteristic analysis when the proposed textile antenna is placed on a high-definition human body model [7], and the results of received power analysis using the ray-tracing method in real environment models.

\section{Analysis of a received power using the ray-tracing method}

Fig. 2 is ray-tracing simulation models that simulated a hospital room, and Fig. 3 (a) shows the result of calculating the received power. The textile antenna in Fig. 1 are used for the transmitting textile antennas (Tx), and a half-wavelength dipole antenna is used for the receiver antenna $(\mathrm{Rx})$. Tx are fixed on the chest and the back of the human body, which height is $1.2 \mathrm{~m}$. 


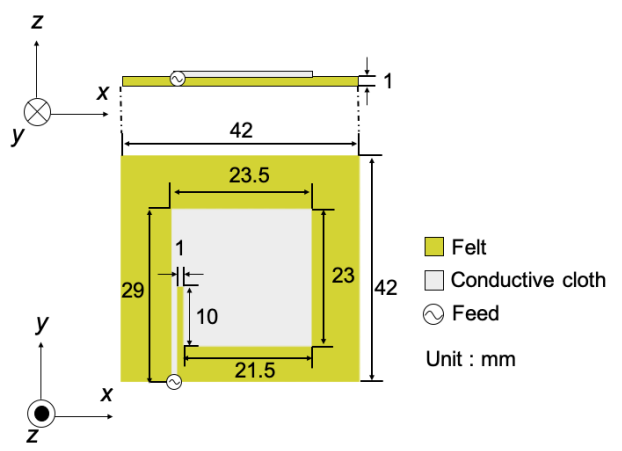

(a) The designed textile antenna

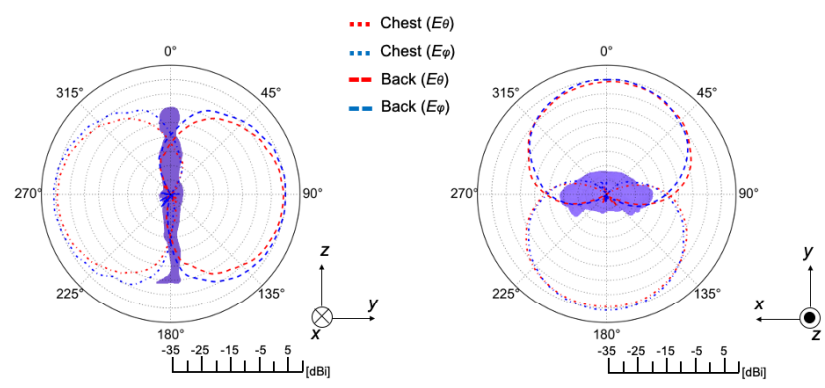

(a) The realized gain of the textile antenna

Fig. 1. The textile antenna and the realized gain

The designed transmitting textile antenna is shown in Fig. 1. In this antenna, using a conductive fabric (conductive cloth/electromagnetic shielding cloth MKKTN 260 manufactured by Tanimura Co., Ltd.) for both a radiating element and a ground plane. A conductivity $\sigma_{e}$ set to $8.29 \times 10^{6} \mathrm{~S} / \mathrm{m}$ for the conductive fabric in numerical simulation. We used a felt textile for a dielectric substrate; we set its relative permittivity to 1.36 .

In the process of designing this antenna, the dimensions of the radiating element at $5.25 \mathrm{GHz}$, the desired frequency, were calculated in the initial stage of the antenna design. We did not use a coaxial power supply from the grounding side, which requires a power supply structure on the back of the antenna. This is because it is assumed that the grounding side of the antenna is used to face the human body. Therefore, we have adopted a method to supply power from the same plane as the radiation elements using MSL (Micro Strip Line). Thus, the resonance frequency was adjusted by adjusting the path length of the current flowing through the radiation element in a right-angled upward direction. The end of the MSL is set near the endpoint of the radiating element. For impedance matching, slits $1 \mathrm{~mm}$ wide and $10 \mathrm{~mm}$ long were made along the MSL. The characteristics of the antenna were evaluated by the FDTD simulation and measurement. As a result, it was confirmed that the present antenna emits two orthogonal polarizations through the single feed point. In addition, it was confirmed that the double polarization is effective in RSSI measurement to respond to the patient's posture change [5].

For the receiver antenna, this is because most wireless LAN access points use dipole or monopole antennas. Therefore, in this numerical analysis, we use a numerical antenna model with characteristics almost similar to those of the actual 


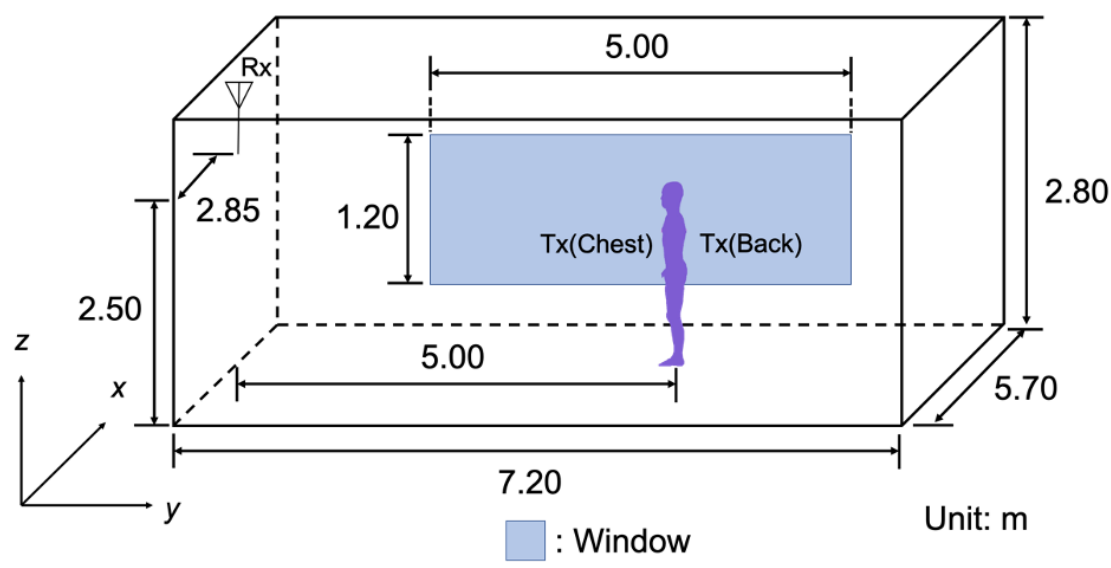

(a) Standing model

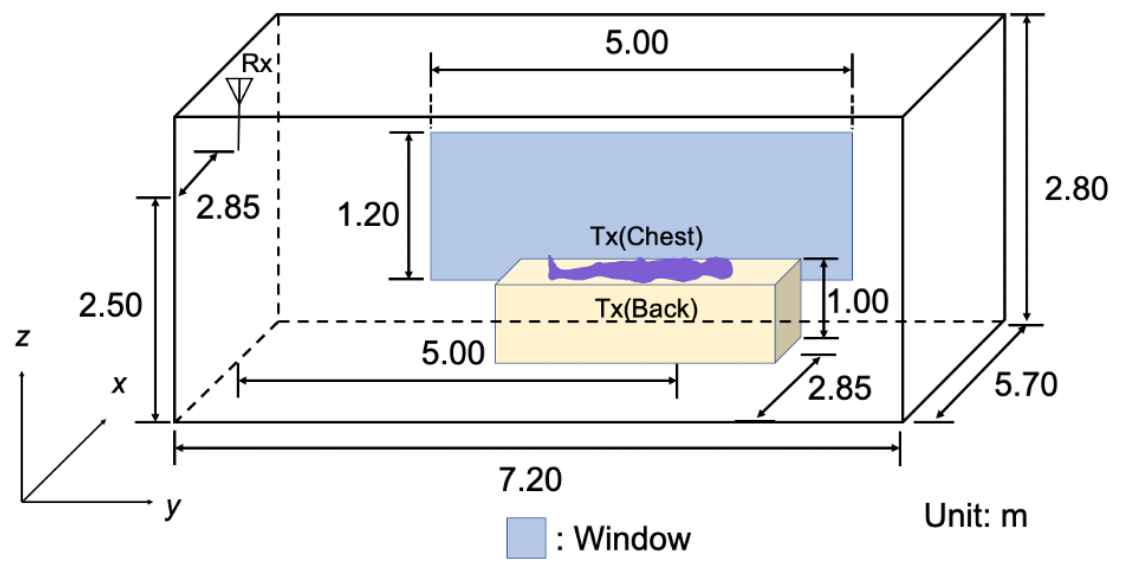

(b) Lying model

Fig. 2. Ray tracing model

of reflections for each wall of $x, y$, and $z$ was set to 3 in all, and the maximum number of diffraction in each direction was set to 1 . In these simulated conditions, the sum of the generated paths results in 340. The number of reflections and the number of passes are the minimum number of times that the analysis results converge in this model. The FDTD program has calculated both Tx and Rx antennas radiation patterns. The values from these radiation patterns have used to calculate the raytracing according to the ray's angle. This received power is defined as the average received power through the receiving antenna port. The received power included all direct, reflected, and diffracted wave paths, and the sum of the ray power has calculated. The total received power from the chest and back antennas when the receiving antenna was fixed at $y=0 \mathrm{~m}$ and the human body with transmitting antennas were moved from 0.0 to $7.0 \mathrm{~m}$ in the $y$ direction is shown in Fig. 3 (a). The total received power was greater than $-70 \mathrm{dBm}$ at all points, and the variation of received power was very small. Furthermore, it was also observed that the received power decayed as it approached the window side. The reason for this is that the reflective and transmission characteristics of concrete and glass are different. The results indicate that diversity can be transmitted using these chest and back antennas for biological monitoring in a real-world environment and that stable communication 


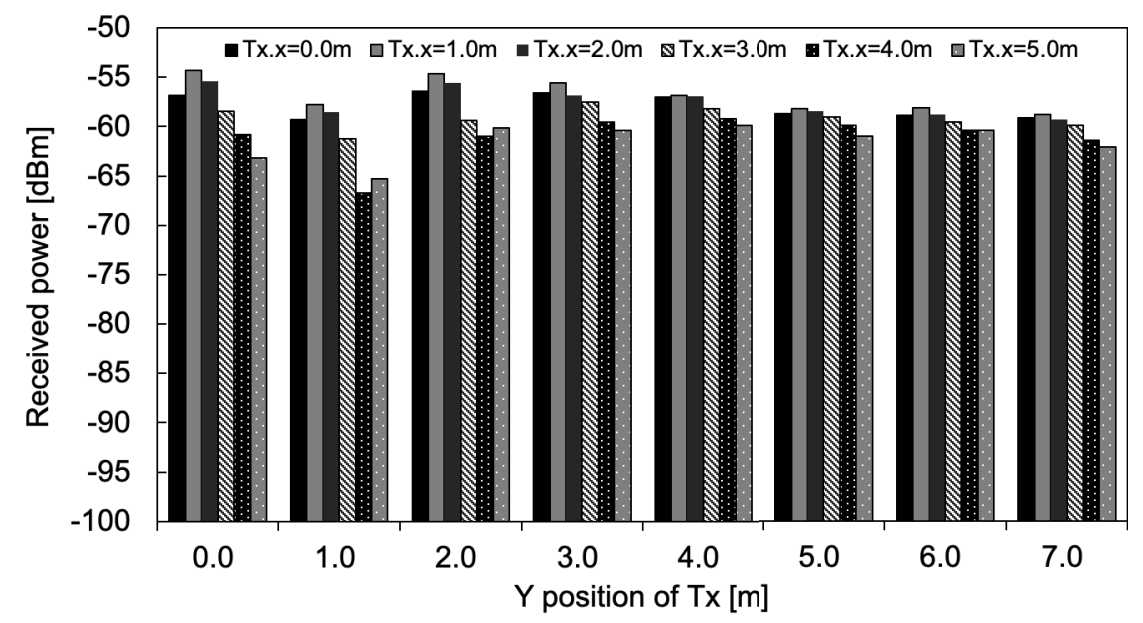

(a) Sum of the received power on $\mathrm{Rx}$ under Tx moved situation



(b) Received power from $\mathrm{Tx}$ (Chest) under Rx moved situation



(c) Received power from Tx(Back) under Rx moved situation

Fig. 3. Calculated received power

is possible.

The results of the received power analysis when the human body is fixed at $d=5.0 \mathrm{~m}$, and the receiving antenna was displaced horizontally $y$ direction from $0 \mathrm{~m}$ to $7.2 \mathrm{~m}$ are shown in Fig. 3 (b) and (c). At the chest antenna, it has been shown that the power can be transmitted at an almost constant rate regardless of the position

๑ IEICE 2020

DOI: 10.1587/comex.2020XBL0116 Received August 23, 2020

Accepted September 15, 2020

Publicized September 30,2020

Copyedited December 1, 2020 
of the receiving antenna. Again, it can be observed that the received power level decays as it approaches the window side. On the dorsal side, the standing position of the human body is $y=5 \mathrm{~m}$, which indicates that almost all points are in the NLOS environment and the received power intensity is low.

Further evaluated the received power when the patient was lying down with the model in Fig. 2 (b). In the model, the bed is made up of PEC (Perfect Electric Conductor) and felt fibers, and these electrical characteristics are set to be the same as in the antenna model. The textile antenna placed on the chest and back of the human model is described in Fig. 1. This is an approach to ensure a reliable wireless connection, regardless of the patient's posture. The results of the received power analysis of the supine model in the supine position are the thoracic antenna is $-59.4 \mathrm{dBm}$, and the dorsal antenna is $-100.6 \mathrm{dBm}$. You can't communicate with the back antenna alone, but it can be seen that it is possible to communicate well enough with the chest antenna. These results shows the importance of using multiple textile antennas in a distributed manner because it is difficult to achieve stable communication with a single textile antenna alone.

\section{Conclusion}

From the results, we can conclude that in a hospital room, when the receiving antenna is a half-wavelength dipole, the textile antenna attached to the chest or back of the human body can provide sufficient received power strength of more than $-70 \mathrm{~dB}$, regardless of the human standing position. By operating these two antennas as a diversity of antennas, it is possible to ensure stable communication. Furthermore, we have found that even when lying down, received power can be provided by at least one textile antenna in the LOS environment. In the future, we plan to analyze the received power in lying down and seated conditions. 